\title{
Sistemas de información en Internet: estudio de un caso
}

\author{
ANTONIO GARCÍA JIMÉNEZ \\ INMACULADA CHACÓN GUTIÉRREZ \\ Departamento de Periodismo Especializado, F. de Cienciasde la Información, \\ Univ. Europea-CEES Tel: 349166478 00. Ext. 219 Fax. 34916168265 \\ E-mail: antonio.garcia@fcp.cin.uem.es \\ ALberto Díaz Esteban \\ Pablo Gervás Gómez Navarro \\ Departamento de Inteligencia Artificial, Escuela Superior \\ de Informática, Univ. Europea-CEES
}

\section{RESUMEN}

Se estudian los sistemas de información existentes en Internet relacionados con el mundo editorial. Se analizan fundamentalmente sistemas en español tomando como punto de partida la evaluación en torno a la personalización y el envío de información a través del correo electrónico. Este análisis se sitúa en un entorno formado por dos marcos de trabajo: por un lado, la evaluación de algunos sistemas representativos con la intención de extraer las características óptimas que deben poseer los servicios de comercialización y de envío de información sobre libros. Por el otro, la extrapolación de la idea de comercialización bibliotecaria a los servicios de venta de libros en la red, ya que una de las ideas que subyacen en este estudio se basa en que las librerías digitales pueden ser analizadas con base en una perspectiva documental y bibliotecaria.

PalabrasClave Sistemæsdeinfomæacón, Inteme, Indstria edtarial, Libreriasdgitales

\section{INFORMATION SYSTEMSON THE INTERNET: A CASE STUDY ANTONIO GARCÍA-JIMÉNEZ, INMACULADA CHACÓ-GUTIÉRREZ, Alberto Díaz-Esteban y Pablo Gervás Gómez-Navarro}

\begin{abstract}
Information systems related to the publishing world are studied with a primary focus on systems in Spanish, taking as a starting point the evaluation of the personalized conveyance of information through E-mail. This analysis lies in a context formed by two frameworks: on one hand, an evaluation of the optimal characteristics exhibited by some representative internet-based book sellers in the conveyance of information on books, and on the other, the extrapolation of the idea of library-like marketing to the internet-based book sellers, since the
\end{abstract}

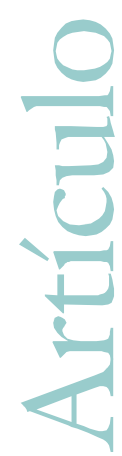

Trabajo recibido el 31 de octubre de 2000 * Trabajo aceptado el 15 de diciembre de 2000 
underlying thrust of this study proposes that digital book stores can be analyzed from documentary and bibliothecary perspectives.

KeyVbrd InfarmationSystems Inteme, PudistingIndstry, Digita Bookstores

\section{INTRODUCCIÓN}

Tas nuevas tecnologías aplicadas al campo de la información están introduciendo $\mathcal{L}_{\text {cambios espectaculares en la organización y en el acceso al conocimiento. La pro- }}$ liferación de sistemas de información basados en técnicas de Inteligencia Artificial, capaces de personalizar el envío de mensajes a través de correo electrónico, obliga a los profesionales de la información a plantearse la necesidad de estudiar cuáles son esos cambios y cómo afectarán a la búsqueda y recuperación de información.

Actualmente existen en Internet numerosos sistemas que envían a sus usuarios mensajes que responden a un perfil definido previamente. Dichos mensajes contienen información sobre fotografías, libros, prensa, etcétera. Las posibilidades implicadas en el contenido del mensaje están abiertas a cualquier tipo de producto, y no se centran únicamente en el envío de informaciones sino también en la comercialización de dichos productos. El presente artículo estudia algunos sistemas existentes en Internet vinculados al envío de información sobre libros y a su comercialización. Se analizarán fundamentalmente sistemas españoles aunque también se han seleccionado algunos sistemas internacionales para poder extrapolar los resultados de la investigación a un marco geográfico más amplio.

Como punto de partida se ha tomado la evaluación que se le realizó a un sistema de envío personalizado de noticias a través del correo electrónico (Díaz Esteban, A. a. 2000). Esta evaluación giraba en torno a Mercurio ${ }^{1}$, un sistema por medio del cual el usuario puede definir sus intereses a través de un perfil que le permite seleccionar noticias o bien mediante las secciones del periódico, o mediante un conjunto determinado de categorías, o por palabras clave. Además permite ponderar la búsqueda y limitar el número de noticias que desea recibir el usuario. En dicha evaluación se analizaron más de 15 sistemas colaterales a Mercurio (normalmente pertenecientes a diarios digitales) existentes en Internet, y se llegó a las siguientes conclusiones (García Jiménez, \&. al. 2000):

* Una de las ideas claves es la personalización. Es importante dotar al usuario de una gama amplia de opciones para que construya su propio perfil de usuario, siempre y cuando éstas sean presentadas de forma clara. Con respecto a las categorías parece aconsejable considerar dos niveles de profundidad (categorías y subcategorías), al mismo tiempo que es conveniente que los sistemas presenten, de forma conjunta, las secciones, categorías y pala-

1 Mercurio: sistema proveedor de contenidos y buscador de información inteligentes. Proyecto financiado por el Ministerio de Industria. Iniciativa ATYCA (TS203/1999). 
bras clave, junto con la posibilidad de que el usuario pueda introducir nuevas categorías. No obstante una gran parte de los sistemas analizados no presenta ninguna forma de categorización y en algunos casos sólo permiten seleccionar las secciones del periódico. En general los sistemas colaterales analizados no personalizan la información, es decir ofrecen poca adaptabilidad para el usuario. Por último se ha observado que es preferible enviar un número no muy elevado de noticias y que el usuario pueda seleccionar los periodos en que desea recibir la información.

- Los sistemas presentan diferentes respuestas en lo que respecta a la satisfacción de las necesidades de información de los usuarios. En efecto, se ha de cuidar la relevancia y efectividad de la información recibida y, al mismo tiempo, se ha de impedir que el usuario reciba información y servicios no solicitados. Es aconsejable que el usuario pueda establecer los niveles de relevancia con los que quiere trabajar en la búsqueda y en la recuperación de noticias.

* Un elemento importante es la posibilidad de acceder a los documentos finales y a los resúmenes de noticias. En efecto, se ha comprobado que el esquema de trabajo [titular; resumen; noticia] es competitivo.

- Otro factor de calidad es la facilidad de acceso y de trabajo con el sistema, lo cual incluye la rapidez de transmisión y la facilidad para poder darse de alta y de baja del servicio. En resumen los sistemas no presentan demasiadas dificultades en su uso.

* Se ha de procurar que el servicio de envío de noticias esté imbricado en una empresa de información de actualidad y que haga posible que el usuario pueda, por ejemplo, acceder a su hemeroteca digital.

* Es recomendable que el sistema presente un diseño de calidad (tipografía, colores, etcétera), sobre todo en lo concerniente a la disposición de cada mensaje. Características que, en general, cumplen todos los servicios.

* La interactividad se convierte en un factor de competitividad. Se recomienda que el usuario pueda ponerse en contacto con el servicio mediante algún responsable para que pueda plantear dudas, realizar sugerencias, etcétera.

Los sistemas de envíos de noticias deberían cumplir estas características. Sin embargo, cuando hablamos de sistemas de comercialización de productos hay que tener en cuenta algunos aspectos específicos aplicables a la utilidad de dichos sistemas dentro del marco de Internet. El objetivo que se debe cumplir es proporcionar al usuario-cliente la mayor cantidad de información en el menor tiempo posible. Por otra parte la forma de presentar la información, ya sea en relación con cuestiones técnicas o en el diseño de la interfaz, son elementos importantes cuando se sabe que el diseño no es un elemento definitivo para el éxito de una estrategia web. 
Como factores positivos resaltan el orden; la estandarización de las interfaces con el usuario; la posibilidad de recoger las intervenciones de los usuarios; la posibilidad de que el cliente encuentre la ruta desde cualquier página pudiendo elegir entre el mayor número de opciones, y, finalmente, la existencia de enlaces predecibles. La prioridad de estos sistemas radica en saber diferenciarse, en presentar nuevas ideas o iniciativas. El objetivo debe ser conseguir una posición preferente mediante una propuesta de valor que sea difícilmente igualable y que proporcione utilidad.

Hay que saber que triunfan los proyectos que les aportan personalización y mayor capacidad de decisión a los clientes Parece que lo más adecuado es usar la información sobre los diferentes segmentos de clientes para presentarles productos y precios personalizados. A través de las preferencias y de la personalización de la oferta se puede captar y mantener la fidelidad de los clientes. La condición de fidelidad depende también de la forma en que se implementen los sistemas para retener al usuario (e-mail gratuito, chats, espacio para su web, servicios de ranking, o bien algún tipo de descuento, de cupón (personalizado) o de sistema de puntos o de promociones), así como del grado de uso, por parte de otros usuarios, que tenga el servicio y la seguridad que proporcione. El cliente debe poder confiar en un servicio en el que obtenga un trato correcto y donde encuentre información sobre los procesos e instrumentos del sistema, así como formas de asesoramiento y de atención a sus objeciones.

En lo que respecta a los precios cabe resaltar que es fundamental la personalización de los precios en función de los hábitos de compra. Para conocer los precios adecuados es conveniente realizar pruebas mediante promociones, sabiendo que no se puede vender a precios superiores a los de los canales tradicionales. Finalmente no conviene olvidar la conveniencia de ajustar las características de los productos informativos a las diferencias de los clientes. Son muchas las dimensiones de trabajo: resolución de imágenes, velocidad de las operaciones, formatos, tiempos, interfaces, diversificación de las características y de la capacidad, amplitud o los soportes (Cornellá, 1999).

\section{EL CONTEXTO}

Este trabajo está vinculado a Perseo ${ }^{2}$. Se trata de un Sistema Automático de Comercialización Personalizada para el sector de las librerías (libros y música) basado en técnicas de análisis del contenido textual, (categorización (Sebastiani, 99), y recuperación (Baeza-Yates and Ribeiro-Neto, 99) de textos), así como en modelado de usuario. El objetivo de Perseo es determinar para cada cliente las novedades que podrían interesarle y enviarle un mensaje o carta personalizado para incentivar su compra y fidelizar al cliente.

Un usuario se registra en el sistema proporcionando una dirección de correo electrónico, un login y un password. A continuación construye un perfil o modelo de

2 Perseo: sistema automático de marketing personalizado. Proyecto financiado por la Comunidad Autónoma de Madrid (Programa Regional de I+D (09/0038/1999)) 
usuario a partir de sus preferencias: cuándo desea recibir novedades, número máximo de ítems por mensaje, desactivación temporal del servicio y delimitación de los intereses del usuario. Estos intereses se pueden definir, conforme con las secciones de la librería Crisol, con base en un conjunto de categorías que son presentadas como sistema alternativo de clasificación (que se corresponden con las categorías de primer nivel de Yahoo España) y con los términos elegidos por el propio usuario.

El sistema también incorpora un sistema de búsqueda que le permite al usuario llevar a cabo búsquedas básicas sobre los distintos artículos de la web de Crisol puesto que puede estar interesado en una información concreta pero no incluida en su perfil. El usuario podrá modificar su modelo sabiendo que el sistema se ejecutará con su perfil con la frecuencia que él mismo haya indicado. El mensaje recibido por el usuario contiene: su nombre, la fecha y, de cada novedad, el título, el autor, su relevancia (en porcentaje), su sinopsis y un enlace a la información completa en la web de Crisol. Al final del mensaje se muestran los intereses que tiene especificados en su perfil en función de las secciones, las categorías y los términos elegidos.

Desde un punto de vista técnico es relevante señalar que la representación de la información se obtiene gracias a la aplicación a la sinopsis de cada producto del modelo del espacio vectorial (Salton, 89). La representación de las categorías se realiza con el texto asociado a las páginas web de las categorías de primer nivel de Yahoo España. Los términos se representan también mediante el modelo del espacio vectorial. Para realizar la selección se categorizan automáticamente las novedades respecto al sistema alternativo de clasificación, y se seleccionan las novedades más relevantes con respecto a los términos elegidos por el usuario. Además los nuevos productos se procesan para confirmar que pertenecen a algunas de las secciones elegidas por el usuario. Finalmente los resultados que se obtienen se integran para obtener un valor final de relevancia.

La evaluación de los sistemas y servicios seleccionados (realizada sobre unas 20 librerías y editoriales, casi todas españolas) se realiza de forma cualitativa a través tanto de una plantilla de trabajo que surge de la adaptación de diferentes trabajos (Slype, 1991; Pastor y Asensi, 1999; Spink et. al., 1998; Barry y Chamber, 1998) como de los criterios que a continuación se exponen:

a) Sistema de categorización: se analiza la existencia de categorías y los diferentes niveles de categorización.

b) Sencillez del manejo: se intenta precisar si el sistema le presenta al usuario alguna dificultad en su utilización.

c) Personalización: se estudia, desde una perspectiva general, si el servicio de información y comercialización ofrece la posibilidad de personalizar la información.

d) El mensaje recibido: se analiza cómo se organizan y estructuran los mensajes que recibe el usuario en su correo electrónico.

e) Acceso al catálogo digital: se estudian los diferentes catálogos que presenta el servicio. También la forma de acceso a los títulos, y el tipo de búsqueda y de visualización de los resultados. 
f) Servicios asociados y de valor añadido: se analizan distintos servicios que puede encontrar el usuario en cada sistema, y que no se sitúan dentro de los servicios estudiados en sentido estricto.

g) Valoración general: después de analizar todos los indicadores de estudio se expone una evaluación general del sistema estudiado.

Una de las ideas que subyacen en este estudio es que nos encontramos ante un conjunto de librerías digitales que pueden ser analizadas con base en un componente documental y bibliotecario. De hecho, desde el prisma bibliotecario, las acciones de comercialización de una biblioteca van dirigidas a los siguientes pasos tanto en los objetivos que deben ser planteados como en los canales que se utilizan (Mañas Moreno, 1999):

a) Establecimiento de objetivos:

* Prestar servicios cuya utilidad para el usuario esté demostrada.

* Lanzamientos al mercado a través de ofertas de productos y servicios informativos con el objetivo de conseguir nuevos clientes

* Se considera un factor relevante que los sistemas presenten mejoras continuas, puesto que unos de los objetivos más importantes se centra en que los usuarios no dejen de utilizar el servicio que se presta.

* Conseguir una mayor frecuencia de uso por parte de los usuarios mediante el mejor aprovechamiento de los recursos informativos que se ofrecen.

* Conocer y mejorar la imagen que se tiene de los servicios, y cambiarla si es necesario.

* Crear una idea en el sector en el que se sitúa, y aportar el concepto de que si se usan adecuadamente los servicios de que se dispone, influirán de forma directa en su trabajo, en su acercamiento a la cultura, etcétera.

b) Canales para la difusión:

* Relaciones públicas: gestionar de forma adecuada las quejas transmitiendo los datos necesarios y creando un clima de confianza abierto y amable.

* Materiales publicitarios escritos:

a) Hojas informativas de carácter general. Se comunican aspectos sobre cómo funciona el servicio: servicios, condiciones de usuario y acceso, reglamento, ordenación y ubicación de fondos, tipos de materiales, etcétera.

b) Hojas informativas de carácter específico.

* Materiales publicitarios audiovisuales: se recomienda un alto uso de imágenes. * Publicidad electrónica:

a) Nivel de contenidos: presentación de la página web, información sobre el Centro, sobre las colecciones y fondos, los recursos, las visitas virtuales, etcétera.

b) Nivel formal: organización de espacios y señalización, presentación atractiva, actualización periódica, estructura clara y sencilla de contenidos, accesibilidad en varios idiomas. 
Formación de usuarios: hay que hacer posible que el usuario pueda formarse gracias al uso del servicio.

\section{DESCRIPCIÓN DE ALGUNOS SERVICIOS}

En este trabajo describimos algunos de los servicios más representativos con miras a que se pueda formar una idea sobre el estado de la cuestión:

\section{Casa del libro (http://www.casadellibro.com)}

* Sistema de categorización: no existe la posibilidad de acotar la suscripción mediante categorías. Sin embargo para la búsqueda de libros el sistema sí ofrece una lista de categorías: literatura, ciencias humanas, artes plásticas, geografía, viajes, gastronomía, medicina, economía y empresas, ciencia y tecnología, informática, libros electrónicos, derecho y ocio, deportes y, finalmente, juegos. A su vez cada una de éstas está dividida en diferentes subcategorías.

* Sencillez del manejo: es un sistema sencillo; su manejo no presenta complicaciones.

* Periodicidad: no le permite al usuario determinar la periodicidad de los mensajes.

* Personalización: no ofrece formas de personalización del sistema, como pudiera ser la acotación por temas o categorías de los mensajes de correo electrónico.

* El mensaje recibido: el servicio consiste en el envío, mediante correo electrónico, de todas las novedades editoriales que vayan surgiendo, así como de las ofertas de la librería.

* Acceso al catálogo digital: presenta diferentes catálogos bajo rúbricas como Losmásvendidosdefición Losmás vendidbs denoficióny SeeciónCasa da Librodonde se encuentran los recomendados por la librería. No contiene un catálogo con todas las obras de las que se dispone.

- Servicios asociados y de valor añadido: tiene una sección en la que se recogen las noticias culturales que se van produciendo y le permite al usuario introducir la dirección de correo electrónico y una clave. Esto evita que el usuario se vea obligado a introducirlos cada vez que realice un pedido. $\mathrm{Al}$ mismo tiempo se puede solicitar a través del correo electrónico información bibliográfica sobre cualquier materia, autor, etcétera. $Y$ en caso de que el usuario no encuentre en la base de datos el libro solicitado, la empresa se compromete a buscarlo en el fondo de la librería. Además, ofrece acceso a chats y a foros literarios.

Valoración general: es un servicio muy completo aunque no personaliza en niveles altos, puesto que no se puede acotar la suscripción por categorías con miras a la recepción de información. 


\section{Libros de E1 Corte Inglés (http://www.elcorteingles.es)}

* Sistema de categorización: ofrece dos niveles. En el primero nos encontramos con 13 temas (bellas artes; ciencias humanas; ciencias naturales; ciencias técnicas; exactas y aplicadas; derecho; diccionarios; economía; historia; idiomas; infantil y juvenil; informática; literatura; tiempo libre) y un segundo nivel de categorización donde no es raro encontrarse con 10 subsecciones para cada uno de los temas anteriores.

* -Sencillez del manejo: desde un punto de vista general el sistema no resulta complejo en su manejo.

* Personalización: no le permite al usuario opciones de personalización.

* El mensaje recibido: no ofrece la posibilidad de recibir e-mail con información pertinente.

* Acceso al catálogo digital: además de la búsqueda por temas, permite buscar por título, por autor, por editorial, por colección, por tema, por idioma y por ISBN. Se accede a la información de cada libro en dos niveles. Existe un formato reducido con el título, el autor, el resumen, la imagen (pequeña) y el precio. El formato ampliado ofrece un mayor número de datos: el título, el autor, el número de edición, la editorial, el número de páginas, un resumen, el ISBN, el precio habitual, el descuento, el precio con oferta y una imagen más grande que la del otro formato. Al mismo tiempo ofrece información sobre la colección en la que se encuentra, la encuadernación, el tamaño y el idioma, lo que le permite al usuario enviar su opinión y conocer libros relacionados.

* Servicios asociados y de valor añadido: el sistema ofrece diferentes novedades (a las que también se puede acceder por tema), una lista con los libros más vendidos y las opiniones sobre alguno de los libros (se trata de críticas realizadas por expertos o por clientes). Al mismo tiempo le ofrece a cada usuario la posibilidad de enviar su propio análisis. Otro servicio interesante es el de las noticias, así como el de la información que hay sobre el sistema de compra y pago.

* Valoración general: la valoración general del sistema es muy positiva, tanto en el acceso al catálogo general como en lo que respecta a la categorización y a los servicios asociados y de valor añadido.

\section{Book on hand (http://www.bookonhand.es)}

Sistema de categorización: presenta tres niveles de categorización donde sólo el primero se compone de 14 categorías (agricultura e industria; arte; catalogación; ciencia y matemáticas; derecho; economía y empresa; educación y psicología; filosofía y religión; geografía e historia; ingeniería y arquitectura; lengua y literatura; medicina y veterinaria; ocio; sociología y política). A continuación hay diversas subsecciones con, a su vez, diversas áreas específicas 
Sencillez del manejo: no representa ningún problema en el momento de su utilización.

* Personalización: desde una perspectiva general no ofrece la posibilidad de personalizar la información.

El mensaje recibido: no ofrece esta posibilidad.

Acceso al catálogo digital: el acceso a los títulos se realiza indicando la sección y la subsección. Permite la búsqueda por editoriales, por autor y por título. Los libros aparecen en la pantalla del ordenador de dos formas: una primera dentro de cada área específica, con su ISBN, el título (junto a la fecha de publicación), el precio y el número de edición, y una segunda en la que se puede ver el autor, la editorial, la edición, la fecha de publicación, la lengua, el precio, la encuadernación, el número de páginas, la colección, el traductor y los puntos pertinentes.

* Servicios asociados y de valor añadido: el usuario puede encontrar un apartado de suggenias(enlaces recomendados), otro de tajjłas(explicación de formas de pago), otro de batique(puntos), otro de notedades promaiones ree mendaciones best-sdler, y además el sistema informa sobre la propia empresa.

* Valoración general: es un sistema aceptable de búsqueda de información. Los tres niveles de categorías ofrecen una forma relevante de búsqueda además de presentar alternativas interesantes en servicios asociados a la compra de libros. También se puede calificar como atractivo.

\section{Bol (http://www.es.bol.com)}

* Sistema de categorización: permite la búsqueda por temas por dos niveles. En el primer nivel se pueden encontrar 22 categorías (arte y diseño; autoayuda y Esoterismo; ciencia y naturaleza; ciencias sociales; deportes; derecho y política; economía y negocios; ensayos y biografías; espectáculos; cine y música; filosofía y religión; gastronomía; cocina y vinos; historia; hogar y familia; informática e internet; ingeniería y tecnologías; libros infantiles; libros juveniles; literatura y novela; medicina; obras de consulta e idiomas; ocio y pasatiempos; viajes y geografía) junto a otro nivel de subcategorías.

- Sencillez del manejo: es relativamente fácil de utilizar.

* Periodicidad: no tiene ninguna periodicidad concreta; esto depende de que el sistema la considere oportuna.

* Personalización: no permite la edición de un perfil de usuario para recibir información sobre las áreas que éste desee. No obstante ofrece la posibilidad de crear una página personal (Mi Bol) que permite al usuario seleccionar los temas (empleando el mismo grupo de categorías y subtemas (uno o varios) o incluso el nombre del autor); y por otro lado ofrece información de la cesta. La personalización se realiza mediante la dirección del correo electrónico. 
* El mensaje recibido: presenta noticias y ofertas, y le ofrece la oportunidad al usuario de darse de baja de la suscripción. Propone algunos enlaces con la página principal, si bien no muestra una interfaz parecida a la del sistema general, e incorpora una lista de novedades y promociones.

* Acceso al catálogo digital: permite el acceso distintos niveles de acceso. La información en Mi Bol ofrece enlaces a especiales y a otros títulos de interés. Es relevante la opción de consultar y conocer los libros más vendidos en un subtema determinado, junto con los datos habituales de cada libro, como puede ser un resumen. De cada libro aparece la siguiente información: título, autor, tiempo de salida del almacén, tipo de encuadernación, editorial, año de publicación y precio e imagen. En un segundo nivel aumenta la imagen y aparece el ISBN además del tema y el subtema al que pertenece el libro.

* Servicios asociados y de valor añadido: enlace a los libros más vendidos, acceso a novedades, a BOL internacional, acceso a libros relacionados, enlace al tema, acceso a la valoración de otros clientes y a la incorporación de la opinión de cada usuario.

* Valoración general: el sistema de categorías es adecuado. La información a través de subcategorías permite establecer un correcto perfil del usuario, aunque la información generada para el correo electrónico es mejorable.

\section{Amazon (http://www.amazon.com)}

* Sistema de categorización: en el dominio de libros ofrece la posibilidad de buscar información a partir de 30 categorías, como pueden ser: Biographies \& Memirs, Business\& Inesting Honor, Nonfiction, Säenee, etcétera. Estas categorías se subdividen en subcategorías hasta alcanzar un tercer nivel.

* Sencillez del manejo: es un sistema sencillo pese al gran número de opciones que tiene. En algunos puntos sería deseable una mayor explicación sobre el sistema.

* Periodicidad: los mensajes de correo electrónico no tienen una periodicidad definida.

* Personalización: permite crear una página personalizada y el sistema realiza recomendaciones de distintos productos. Al acceder al sistema el usuario se encuentra con el servicio Nenfor Yau con un saludo al usuario (donde aparece el nombre de éste). El factor de personalización también se destaca en los mensajes de correo electrónico, en los que aparece el nombre del cliente.

* El mensaje recibido: comienza con un WedametoNenforYau Divide la información en NewRdes Toselesy PurchaseCirdescon respecto a libros y a otros tipos de artículos. Por otra parte desde el mensaje se puede explorar la página de Amazon en la que se podrá acceder a más información. 
Acceso al catálogo digital: los libros se ven en un primer nivel con una imagen pequeña, el título, el autor, el precio, un resumen, información para la compra e información relacionada. El segundo nivel presenta el título, el autor, el precio, el ahorro que se produce, la disponibilidad del libro, una imagen, críticas de usuarios y los libros similares por temas o categorías. En un tercer nivel aparece la fecha, la posición en cuanto a ventas asociadas, una imagen de mayor tamaño, los libros comprados por otras personas que también adquirieron ese libro, además de opciones tales como la posibilidad de explorar el libro (tabla de contenidos, un repaso a comentarios críticos tanto de entendidos como de clientes, más información sobre el autor, y la posibilidad de compartir ideas sobre el libro mediante el correo electrónico). Por último, la búsqueda se realiza a través de: autor, título, tema, ISBN, editorial, fecha de publicación, formato, edad del lector y lengua (estos resultados se pueden ordenar por prioridades).

* Servicios asociados y de valor añadido: el análisis de Amazon ha de hacerse reconociendo que se trata de una auténtica tienda: destaca las novedades, enlaza con entrevistas, con enlaces recomendados, con noticias y con bestsellers.

Valoración general: es un muy buen sistema y muy completo que ofrece alta o media calidad en cada uno de los anteriores apartados. En realidad se ha convertido en una tienda que supera el concepto de librería.

\section{Editorial Planeta (http://www.editorial.planeta.es)}

* Sistema de categorización: los libros pueden buscarse por título, autor y/o colección, pero el sistema no ofrece la posibilidad de acotar la búsqueda por categorías.

* Sencillez del manejo: es fácil de utilizar.

* Periodicidad: no tiene servicio de información de novedades editoriales a través de e-mail

Acceso al catálogo digital: tiene diferentes catálogos, uno con las novedades, otro con los libros más vendidos y otro con las reediciones. No dispone, sin embargo, de un catálogo con todas las obras editadas en Planeta.

Servicios asociados y de valor añadido: permite el acceso a información sobre premios y concursos literarios (ganadores de este año y de ediciones anteriores, bases del concurso, etcétera) y a una sinopsis de cada obra o a información sobre su autor. Además incorpora tanto una sala de lectura, donde se pueden leer fragmentos de algunas obras a través de la red, como diferentes foros, que vienen a ser una sección por la que se puede participar en debates literarios. 
* Valoración general: es un sistema muy completo aunque no permite la compra de los libros sin previa contraseña, tras lo cual, además, se puede acceder a noticias literarias e imágenes de calidad.

\section{Editorial McGraw-Hill (http://www.mcgraw-hill.es)}

* Sistema de categorización: ofrece tres niveles de categorías, en función de sus series (informática; ingeniería; economía/empresa; educación secundaria; ciencias de la salud; ciencias sociales; ciencias y matemáticas; ciencias jurídicas; CD-ROM; novedades; colecciones/series (ciencias de la salud; ciencias jurídicas; ciencias/matemáticas; economía/empresa; informática; ingeniería; otros libros). En el segundo nivel el usuario se encuentra con un alto número de subcategorías, que a su vez están divididas en otras.

* Sencillez del manejo: es un sistema moderadamente sencillo en el momento de su uso.

* Personalización: este sistema no ofrece opciones de personalización salvo en el apartado de Universidad, que incluye en función de determinas áreas de interés, un servicio de información bibliográfica personalizada gracias al correo electrónico.

* Acceso al catálogo digital: fundamentalmente se realiza por categorías. El acceso a los datos de cada libro se realiza mediante dos niveles: el formato abreviado, que incluye el título, el autor y el ISBN; y el formato ampliado, que presenta el título, el autor, el ISBN, la fecha de publicación, la edición, el número de páginas, el formato (tamaño), el tipo de cubierta, el número de volúmenes, el nivel (p. ej. profesional), el precio, un resumen y las distintas partes de la monografía.

* Servicios asociados y de valor añadido: el usuario se puede encontrar noticias; novedades, con un enlace a las divisiones editoriales (algunas con dirección de correo electrónico); un enlace a nuestros dqpositarios, con dros enlaces y con otros en el que se explica cómo adquirir los libros.

* Valoración general: tomando en consideración que se trata de una editorial estamos ante un sistema aceptable.

\section{Servicio Buscaproductos (http://www.buscaproductos.com)}

* Sistema de categorización: no presenta un sistema de categorías. La búsqueda se hace por título y/o autor.

* Sencillez del manejo: es un sistema fácil de utilizar.

* Mensaje recibido: no tiene servicio de información de las novedades editoriales a través de e-mail.

* Acceso al catálogo digital: no ofrece catálogos de ningún tipo.

* Servicios asociados y de valor añadido: ofrece la posibilidad de acotar la búsqueda sólo en tiendas en español y/o sólo en tiendas con opción a regalo (aquellas que permiten indicar en qué fecha debe entregar el producto 
comprado junto a una tarjeta personalizada de felicitación). A su vez el usuario puede ordenar los resultados obtenidos por orden alfabético o numérico, y en función del título, el precio y el autor. En realidad es factible precisar más la búsqueda por los campos correspondientes y se muestran aquellos que cumplan con esas condiciones.

* Valoración general: es un buen sistema orientado a la búsqueda general de libros y permiten hacer uso de todos los recursos que hay en la red para su posterior compra.

\section{CONCLUSIONES: TENDENCIAS Y LÍNEAS DE TRABAJO}

A continuación, exponemos las principales conclusiones obtenidas al revisar de los servicios analizados; cabe tomar en cuenta que estos resultados son orientativos y genéricos. Además este estudio se centra fundamentalmente en los sistemas de información y no tanto en los servicios de envío de correo electrónico, dadas las características de los sistemas:

a) Los valores que están más cercanos a la calidad son los que se vinculan a la búsqueda, y más en concreto, al nivel de detalle, hecho que se demuestra cuando un sistema, por ejemplo, permite especificar la banda de edad del usuario. Al mismo tiempo los sistemas más avanzados resaltan la personalización gracias a la suscripción al servicio de envío de información por correo electrónico, o a la posibilidad de crear una página personalizada. Suelen, por otra parte, ofrecer servicios de valor añadido como son el acceso a páginas literarias, a novedades, a catálogos especializados, etcétera. Por otra parte la calidad también va de la mano de la sencillez. En general ninguno de los sistemas supone una gran dificultad de manejo, aunque se puede afirmar que una gran cantidad de opciones representa un aumento en la complejidad del sistema. Por otra parte cuando se hace referencia a una menor calidad, en realidad, se mencionan cuestiones como la dificultad de acceso a los catálogos, la ausencia de un servicio de suscripción, la simplicidad en los servicios, la ausencia de enlaces de interés, etcétera.

b) En lo que se refiere a la personalización, y si se aplica un prisma global, se observa que ninguno de los sistemas permite personalizar los resultados, ya sea en el mensaje enviado al usuario (aquellos que lo hacen) o en las búsquedas que pudieran realizarse en la propia página web. En este caso destacan en mayor medida Bol (donde el usuario también puede seleccionar temas, subtemas e incluso autores), y Amazon, puesto que permite generar páginas personalizadas. Así, se puede confirmar que la información y los servicios no se despliegan en función de los diferentes usuarios, cosa que se traduce en elementos formales y de contenido.

c) En la recuperación y búsqueda de información destaca el hecho de que las posibilidades de búsqueda de libros no difieren demasiado de unos sistemas a otros. Así, hay servicios en los que la búsqueda se puede acotar además de por catego- 
rías, por el precio y por el intervalo de edad al que debe ir dirigido el libro. Lo habitual es que los usuarios accedan a un servicio de búsqueda en el que recuperan información a través del título, ISBN, tema, autor, fecha, etcétera, aunque es difícil que le permitan al usuario que presente o modifique sus criterios de búsqueda. No obstante, y pese a que los sistemas detectan las novedades existentes y no exigen demasiado esfuerzo, la recuperación de información se enfrenta con varias limitaciones, como la imposibilidad de organizar los resultados en función de un criterio determinado, por ejemplo el de relevancia. Por otra parte la pertinencia y relevancia de la información sobre cada libro se ha determinado a partir de diferentes cuestiones: si existe redundancia en la información; si la información ofrecida es suficiente para determinar la compra de un libro; si se ofrece información sobre el género o el tipo de libro; si no hay datos innecesarios; si se adapta al tipo y perfil de usuario; si se presenta información complementaria; si se presenta en la ubicación esperada; si la información ofrecida presenta la perspectiva, la profundidad y el planteamiento oportuno; si la información viene organizada adecuadamente o si plantea documentos relacionados; y si el libro es considerado relevante o si proporciona los suficientes datos para motivar el interés y su compra. Lo que se ha podido comprobar es que la mayoría de los sistemas no proporcionan datos sobre el género o el tipo de libro y que tampoco proporcionan información complementaria.

d) Todos los sistemas, en mayor o menor medida, se presentan de forma atractiva para los usuarios, y en algunos casos esto se vincula a la sencillez. Es evidente que los componentes gráficos pueden provocar un nivel alto de satisfacción. A esto hay que añadir la facilidad de uso como cualidad que llega a todos los servicios estudiados.

e) La interactividad se puede medir con base en la posibilidad de comunicación con el sistema, no sólo para la mejora del sistema sino para aumentar las posibilidades de búsqueda. A su vez la interactividad tiene que ver con la capacidad para que los usuarios o bien puedan enviar su opinión sobre los libros o bien accedan a la opinión de otros clientes o incluso de críticos. Al mismo tiempo los servicios cuentan con elementos de ayuda para el usuario, tales como una introducción o como los tutoriales.

f) La presentación de los resultados es similar en todos los sistemas. En diversos casos los servicios ofrecen varios niveles de detalle en la información, y en muchos otros dan datos suficientes para la compra. En este apartado destacamos Buscaproductos, porque ofrece un cuadro con el autor, el título, la editorial y el precio, más el tiempo que transcurrirá hasta la recepción del libro y las tiendas en las que se vende cada uno de los libros encontrados.

g) La mayoría de los sistemas presentan un sistema de categorización, aunque en la mayoría de los casos no se conectan con el envío de información mediante correo electrónico sino con la búsqueda de libros dentro del propio sistema. Si hacemos un repaso global, cuando los sistemas tienen categorías se observa que casi siem- 
pre los libros tienen que ver con la categoría suscrita, que no se solapan las categorías y que éstas parecen las adecuadas.

A modo de resumen, las características que conviene tomar en consideración como líneas de actuación más importantes son las siguientes: en primer lugar resulta trascendente la amplitud y el nivel de detalle en la categorización (se prefiere la existencia de dos o tres niveles de categorización), así como la existencia de, además de los genéricos, diferentes catálogos del tipo "Los más vendidos", "Los Recomendados", "Ofertas", etcétera. En segundo lugar es muy importante que el servicio proporcione la posibilidad de definir el perfil del usuario para dirigir los resultados de las búsquedas o para perfeccionar la recepción de información mediante correo electrónico sobre, como mínimo, las novedades editoriales. De esta manera la suscripción, acotada por materias permitiría que el usuario recibiera información de interés.

Por otra parte también resulta importante la existencia de un apartado de ayuda, sobre todo en lo relacionado con la búsqueda, lo que hace posible que el usuario se ponga en contacto con la librería o editorial virtual para preguntar por algún libro no encontrado o para solicitar cualquier información. En definitiva un sitio web se valora de manera más positiva cuando le ofrece mayor capacidad de decisión al usuario y cuando le ofrece más servicios (existencia de una sección de noticias sobre los últimos eventos culturales; acceso a páginas e información relacionadas; opción de incorporar o acceder a resúmenes y críticas de otros usuarios y/o de críticos especializados; posibilidad de organizar los resultados de las búsquedas en función del título, autor, precio, etcétera).

\section{BIBLIOGRAFÍA}

Baeza-Yates, Ricardo and Ribeiro-Neto, Berthier [1999], Mo demInformation Renieal, New York, ACM Press Books.

Barry, Carol L. and Schamber, Linda (1998), "User's criteria for relevance evaluation: a cross-situational comparison", InformationProwsing\& Management, vol. 34, nº 2/3, pp. 219-236.

Cornellá, A. (1999). "Qué funciona en Internet", Extrant, mensaje 472.

Díaz Esteban, A.; Gervás Gómez-Navarro, P. and García Jiménez, A. (2000), "Evaluating a User-Model Based Personalisation Architecture for Digital News Services", Procedingsofthe Fourth Europeen Confereneon Reserch and Advanced Teetholog for Digital Libraies (ECDL'2000), Lisbon, Springer, p. 259268. 
García Jiménez, A.; Chacón Gutiérrez, I.; Díaz Esteban, A.; Gervás Gómez-Navarro, P.; (2000). "Nuevos sistemas de información: tendencias y evaluación”, CuademosdeDoumenta iónMultimela, no 9 , En:

http://www.ucm./info/multidoc/muldico/revista/num9/ prensa/jime-chacon.htm

Mañas Moreno, José Eugenio (1999). "Marketing y difusión de servicios en una biblioteca universitaria del próximo milenio", 7esJamades Catalanes deDoumentadó(Les biblidtequesi es centresdedoumentaciónal sedeXXI: pesadaudelascieatdelainformacio), 4-6 de noviembre de 1999, Barcelona, Collegi oficial de bibliotecaris-documentalistes de Catalunya, p. 73-83.

Pastor, J. A. y Asensi, V. [1999], "Un modelo para la Evaluación de Interfaces en Sistemas de Recuperación de Información", AdasIV CongesoIskoEspañaEconsid99, Granada, Isko-España, Facultad de Biblioteconomía y Documentación, pp. 401-409.

Salton, G. [1989], Automatic Text Proessing the transfomation, analysisandretrieval finfomationbycomputer, Addison Wesley.

Sebastiani, F. [1999], "A Tutorial on Automated Text Categorisation”, ProceeingsoftheFirstArgentinenSymposiumanArtifical Inteligence(ASAI-99).

Slype, Georges van (1991), Loslengrajesdbamentalesdeindizacón: concepión, construrciónyutilizaciónenlossistemasdoumentales Madrid; Salamanca, Fundación Germán Sánchez Ruipérez; Pirámide.

Spink, Amanda; Howard, Greisdorf and Bateman, Judy (1998), "From highly relevant to not relevant: examining different regions of relevance", Infomation Processing \& Managment, vol. $34, n^{\circ} 5$, pp. 599-621. 